\title{
Mentoring, coaching and action learning: Interventions in a national clinical leadership development programme
}

\author{
McNamara MS, Fealy GM, Casey M, O’Connor T, Patton D, Doyle L, Quinlan C
}

\begin{abstract}
Aims and objectives

The study reported here was part of a larger study that evaluated a national clinical leadership development programme in Ireland. The aim of the present study was to evaluate mentoring, coaching and action learning interventions used to develop nurses' and midwives' clinical leadership competencies. The objective was to describe the programme participants' experiences of the interventions.
\end{abstract}

\section{Background}

Mentoring, coaching and action learning are effective interventions in clinical leadership development and were used in a new national clinical leadership development programme, introduced in Ireland in 2011. An evaluation of the programme focused on how participants experienced the interventions.

\section{Design}

This evaluation study was involved a qualitative design, using multiple data sources and multiple data collection methods.

\section{Methods}

Methods used to generate data on participant experiences of individual interventions, included focus groups, individual interviews and non-participant observation. Seventy 
participants, who included fifty programme participants and those providing the interventions, contributed to the data collection.

\section{Results}

Mentoring, coaching and action learning were positively experienced by participants and contributed to the development of clinical leadership competencies, as attested to by the programme participants and intervention facilitators.

\section{Conclusions}

The use of interventions that are action oriented and focused on service development, such as mentoring, coaching and action learning should be supported in clinical leadership development programmes. Being quite different to short attendance courses, these interventions require longer-term commitment on the part of both individuals and their organisations.

\section{Relevance for clinical practice}

In using mentoring, coaching and action learning interventions, the focus should be on each participant's current role and everyday practice and on helping the participant to develop and demonstrate clinical leadership skills in these contexts.

\section{Keywords}

Clinical leadership, mentoring, coaching, action learning, intervention, programme, evaluation 


\section{What does this paper contribute to the wider global clinical community?}

- Clinical leadership development is an ongoing process that takes place over time and in context and therefore clinical leader competencies are best developed through interventions that address individual leader needs as they relate to role performance and service improvement.

- Interventions that are experiential, action oriented and focused on service improvement, such as mentoring, coaching and action learning, should be incorporated into clinical leadership development programmes.

- By assisting nurses and midwives to develop and demonstrate clinical leadership competencies in context, experiential and action-oriented interventions may be preferable to once-off short attendance courses, but they require long-term commitment from individuals and their organisations.

\section{Introduction}

In 2010, the Office of the Nursing and Midwifery Services Director (ONMSD) in Ireland introduced the National Clinical Leadership Development Framework for clinical leadership development in nursing and midwifery (Health Service Executive (HSE) 2012). Integral to the Framework is the Clinical Leader Development Pathway, a developmental process that enables nurses and midwives to gain access to the Framework at the level appropriate to their clinical leadership development needs. Unlike once-off attendance programmes, the Pathway constitutes an ongoing process of leadership development that takes place over several weeks 
and months. The Framework and associated Pathway were piloted among fifty nurses and midwives, representing all grades, at seven sites in Cork city from July to December 2011.

The programme was based on the Royal College of Nursing $(\mathrm{RCN})$ Clinical Leadership Programme, which is a one-year experiential programme that aims to develop nurses' and midwives' transformational leadership behaviours (Lunn et al. 2008; RCN 1997). The material resources that supported the Irish programme, including a learning resource pack, were developed by the RCN and, like the RCN programme, the Pathway element linked participant competence development to service improvement. The Irish programme identified seven core clinical leadership competencies to be developed: self-awareness, advocacy and empowerment, decision making, communication, quality and safety, team work, and clinical excellence. For the purpose of the programme pilot, participants were expected to attain a minimum of two competencies. Accordingly, the programme pilot was run over a period of six months at the seven pilot sites.

A range of interventions was provided to assist participants to develop the two competencies within the six-month timeframe and the competencies were selected on the basis of an individual assessment of leadership development need. The assessment was conducted by each Pathway participant in consultation with his/her line manager, using an Assessment and Development Tool designed specifically for the leadership development programme. The purpose of the tool was to assist the Pathway participant and his/her line manager in deciding priorities for leadership development and how these would be linked to the service in which the participant worked. The tool was based on a series of behavioural indicators for each competence, and it yielded a participant score for each competence; the score, in turn, 
indicated development need in each competence and provided the basis for selecting appropriate interventions.

Interventions available to the participants included mentoring, coaching, action learning sets and competence-specific workshops. The facilitators providing the interventions were senior experienced nurses and midwives in clinical practice, service management, practice development or continuing education. Mentors were internal to the HSE organisation in which they mentored and were trained in mentoring by members of the programme project team. Coaches were drawn from other external HSE organisations and were trained by coaching specialists and each coach was contracted to provide an agreed number of coaching sessions. The coaching process also involved regular tri-partite meetings between the coach, coachee and the coachee's line manager, with the aim of agreeing priorities for service improvement and reviewing progress in leadership development. Training for each intervention facilitator was supplemented by additional resources, such as a coaching reference manual and a mentor guide.

A team of researchers from University College Dublin was commissioned by the ONMSD to evaluate the programme at the seven pilot sites, with reference to resources and supports, participant experiences and short-term individual and service-level outcomes (****2012). The team included five nursing academics with prior experience in researching and teaching in the areas of clinical leadership and staff development and one head of staff development at a large tertiary hospital. None of the research team members were known to the participants. The findings relating to the participants' experiences of the leadership development interventions are the focus of this paper. 


\section{Background}

Interventions used for leadership development include 360-degree feedback, coaching, mentoring, networking, job assignments and action learning (Day 2001). Such experiential interventions have received wide support in the literature on leadership development (Proctor-Thompson 2008); mentoring is especially effective for leadership development in context (Day 2001), but requires considerable training in its proper use (Bush et al. 2007). Informal mentoring has been shown to be more beneficial than formal mentoring (Day 2001).

Coaching is advocated as a way of developing clinical leadership skills in context, by placing individuals into real situations in which they experience at first hand the unique problems that occur in particular clinical settings (Byrne 2007). Aimed at improving leadership skills or solving particular problems, it is an ongoing process rather than a discrete event (Day 2001) Coaching is both action and learning oriented, focusing on the individual's personal and professional goals and intrapersonal and interpersonal skills, such as self-management and interpersonal communication (Byrne 2007). Reid Ponte et al. (2006) reported that nurses who experienced coaching found it effective in helping them to identify and correct behaviours that hampered their performance, thereby improving their individual and organisational effectiveness.

Leonard and Lang (2010) reported that the use of action learning as a competence-based intervention for leader development significantly contributing to participants' development as leaders, including the development of specific critical competencies, like communication, team building and decisiveness. These authors concluded that action learning was effective due to its efficiency, its focus on particular individually-relevant leadership skills and its 
focus on real-world practice problems and actionable solutions. Leonard and Lang (2010) also concluded that coaching and action learning were more strongly effective on outcomes like insight, skill development and real-world practice, than more traditional leadership development approaches, such as taught attendance programmes.

Evaluation studies of clinical leadership development programmes have indicated their effectiveness in achieving both individual and service-level outcomes. Studies evaluating the RCN Clinical Leadership Programme have reported service improvements in areas like infection control and patient privacy (Large et al. 2005) and enhancement of leadership skills leading to novel ways of delivering care (Lunn et al. 2008). Stoddart et al. (2012) reported evidence that the Leading Better Care programme resulted in improved leader confidence and team performance and quality improvement.

\section{Methods}

\section{Data collection}

Data were collected from the fifty Pathway participants, their coaches, mentors, actionlearning set (ALS) facilitators, line managers and programme coordinators, resulting in seventy participant contributions. Several complementary qualitative data collection methods were deployed to generate data on programme participants' experiences, including a total of twelve focus groups among the Pathway participants, mentors, ALS facilitators, line managers, service managers, coaches and programme coordinators. The coaches and mentors also provided written comments and reflections that informed the study data. Three individual interviews were conducted. In addition, non-participant observation was used to observe and evaluate a single, purposively selected, action learning set. This method was 
used for process-level evaluation and to provide complementary data on experiences of action learning.

All participants were purposively selected and data collection was conducted over the final six weeks of the six-month pilot. All data collection events were conducted on the basis of a topic guide. Each focus group was facilitated by a moderator assisted by an observer (Krueger \& Casey 2009, Barbour 2007), with discussion in each group directed toward the participants' experiences of the programme and its interventions, either as recipients or facilitators. The observation of the action learning set was conducted with the prior consent of the ALS facilitator and participants and data were recorded as field notes and compiled as a narrative report.

The qualitative data collection procedures were reviewed and approved by the researchers' institutional human research ethics committee. Each participant gave written informed consent prior to participating in a data collection event. Participant anonymity and confidentiality were assured, with reference to the collection, handling and reporting of study data.

\section{Analysis}

Most data collection events lasted from between 45-60 minutes. Data saturation was not a consideration, since it was determined that all participants should be provided with the opportunity to contribute to the data collection. Focus groups and interviews were transcribed verbatim and anonymised and thematic content analysis was performed as a way of organising, analysing and presenting the data. This involved a multi-stage approach adopted from grounded theory (Glaser and Strauss 1967) and from other published works on thematic 
content analysis (Berg 1989, Newell and Burnard 2006), and included note-taking and memowriting, listening to the audio recordings and reading the transcripts.

NVivo 9 software was used to aid data handling and two members of the research team coded the data. In the process, nodes were collapsed together to permit common patterns and themes to emerge, rather than imposing pre-determined themes, and these were grouped into preliminary higher-order categories. The process also involved reaching consensus on categories, allocating category and detail codes, merging and re-allocating details and finally collating the data for presentation. Given the number of data collection events and the multiplicity of participants, the findings were not subjected to participant confirmation.

\section{Results}

Data collection yielded rich narrative accounts of the experiences of Pathway participants and facilitators of interventions. Participant experiences of the interventions were accounted for in one broad category entitled 'experiencing the Pathway process.' This category yielded three themes that describe participant experiences of the three specific interventions, namely: 'experiencing mentoring' 'experiencing coaching' and 'experiencing action learning'. For the purpose of reporting the findings, the nurses and midwives who completed the programme pilot are referred to as 'Pathway participants' and other study participants, such as mentors and coaches, and are otherwise specified.

\section{Experiencing mentoring}

Pathway participants spoke in positive terms about the mentoring intervention. A typical comment was: "My mentoring was great, I was very happy with it." Some spoke about how 
the mentor acted as a motivator. One stated: "my mentor pushed me and she evaluated me and she challenged me" and another said: "she hasn't given me the answers, [but] she has really made me think and she has dangled the carrot for me to bite, but yet guided and supported me all the way." Others spoke of the qualities of their mentors, as the following selected short extracts show: "she is so motivated"; "my mentor is ... all about what to do for me"; "I can discuss anything with her, [and] she is always there". Some spoke about how their mentor seemed to be tuned into their leadership development needs: "Even though we don't work together, [my mentor] just has much more insight into what all this [is] about"; "my mentor has been ... very much focusing on the need to develop myself and the service being developed as a by-product of that."

Pathway participants viewed mentoring as an intervention suitable for enabling individuals to clarify and examine their clinical leadership development needs in the context of their professional role: "it is the mentor who has worked through it with you." Some Pathway participants spoke of the value of the one-to-one arrangement that mentoring involved: "I like to sit down and discuss one to one and that works very well." Others spoke about the importance of having "the right person" as a mentor and about the importance of a good mentor-mentee relationship to the success of the intervention: "it is really a personal thing that you get on well with your mentor and that they have the same vision as you."

Not all experiences of mentoring were positive. One Pathway participant saw greater merit in having a mentoring relationship with a colleague than with an assigned mentor:

I find that my skills are developed more with the people I am working more closely with and that I admire so much, I suppose, that they are my role models ... It would 
have been great [to be mentored by someone with whom I work] (Pathway participant).

One Pathway participant remarked on her experience of feeling like a "puppet on a string" in the mentoring relationship and commented: "I felt it reined me in a little bit." Another observed that the arrangement was somewhat open-ended, in terms of time commitment: "They had nothing to say that my mentor should give me 10 hours between now and Christmas ... There is nothing set to say you need this amount of time, at least to achieve a competence." Another concluded that mentoring was chiefly concerned with "personal development rather than actually looking at service needs."

Mentors themselves spoke about their experiences of mentoring, including how they had assisted their mentee to develop: "I think the mentorship has been hugely beneficial to the mentees and I think it gives them the opportunity in a safe environment to think out a lot of solutions themselves." One mentor spoke of her understanding of the role as "giving the tools, showing the way, signposting what was available for them to do." Another described the importance of the mentee leading the process:

You are there to mentor, to give guidance and to sign post, if the mentee wishes to or not maintain their [learning and development] portfolio at the rate that maybe the guidance suggest, then again it is up to the mentee. It should be very mentee driven because that is their responsibility, certainly not the mentors.

Some mentors used the mentee's learning and development portfolio in a deliberate way to guide the mentoring process, as one mentor stated: "[we] focused very much on the portfolio 
as well and she kept it very well up to date and ... this was a very good way of getting to know [her]." Another similarly spoke about the use of the portfolio to guide mentoring activities:

We used the portfolio from the very beginning and we used it through most of our meetings ... we went back through it and we looked to see if there were any points that needed clarification or a bit more mentoring on ... [and] it gave us something to focus on (Mentor).

Mentors spoke about the sense of limited time to achieve the required two competencies:

So some things have been achieved, other things will take longer to achieve for competence and for, I suppose, to the indicators within the competence to be achieved (Mentor)

Others saw the time constraint as relating to the development of service initiatives, as the following two extracts illustrate:

She has everything set and the ground work done, but it will take a lot longer to actually carry out (Mentor).

My mentee had the impression I suppose that the project had to be done by December ... but inevitably these projects would take longer, particularly if it is around practice changing (Mentor). 
As a normal part of the mentoring process, mentors held self-monitoring and mutual support meetings, and arising from these meetings, mentors proffered observations and suggestions for good mentoring:

Clarity is essential in relation to all aspects of mentoring and both the mentor and the mentee are required to maintain confidentiality and be honest in the process.

The mentor needs to be self-aware and to listen effectively in the mentoring session, in order to ensure that what is being said is heard, and the mentee also needs to listen effectively.

The mentor should aim to balance the levels of challenge and support in the mentoring process.

The ongoing communication between the mentee, mentor and line manager should be led by the mentee.

Additional suggestions from the mentor group included the need for tripartite meetings between mentor, mentee and line managers and the need for flexibility in selecting clinical leadership competencies to reflect both individual and service needs.

\section{Experiencing coaching}

This theme describes participants' experiences of the coaching intervention. The coaching process was described by one coach, who presented written reflections on her experience:

Progress in competence development was enabled by exploring the coachee's current position in relation to a competence and the position [that] they would have attained if more clinical indicators of competence had been achieved, and to map out a route 
between these two points. The route involved addressing many levels of learning to include finding new information, brainstorming actions, deconstruction beliefs, values, attitudes, ways of working and engagement that prevent action and competence growth, and replace them with processes and ways of being as a professional that enhance competence and its application in the clinical field (Coach).

During the coaches' focus group, one coach explained how coaching was linked to the participant's assessment and development plan and how the coaching contract also figured in the process:

The contract ... almost takes up where the assessment and development tool finishes ... part of the role during the contracting meeting is to actually work through what that means so you do continue to some degree part of that assessment and it is really, really important, obviously, that the contracting meeting is very comprehensive ... and part of the contract is about finding out, making all of that explicit and also maybe doing a complete reality check ... about what is possible as well (Coach).

Pathway participants described their experiences of being coached. One spoke about her understanding of the respective roles of the coach and coachee:

It is very much your own agenda and you set the tone for the meeting and she is very challenging as well ... but she won't give you any answers [and] it is very much up to you to find solutions (Pathway participant).

A senior nurse similarly recognised her role as coachee: 
It is not always like "oh the coach is fabulous and she is going to sort everything out"; coaching isn't about that ... coaching is about you deciding what you are doing and they working along with you ... coaching is you, but they are working along with what you are doing (Pathway participant).

Pathway participants spoke of the how the coaching style contributed to their development: "[Coaching] is making you think about it in a different way"; "[The coach] makes you focus more [on] the one thing." The fact that coaching arrangements were "far more structured" than those for mentoring was also seen as beneficial: "You are going off site because there is (sic) interruptions ... and at least when you leave the hospital you cannot worry about anything." For one coachee, choosing the coaching intervention was based on her sense of needing to have "someone who is outside [my work]". Another expressed "amazement of having a sacred space to actually discuss real issues." One nurse spoke about how the coaching intervention had helped her with communication:

I am [now] just more aware of how I am communicating and who I am communicating to and from; that aspect of it has been good ... because I am very conscious of [communication] ... and how do you respond to [everyday problems]' (Pathway participant).

One coach saw the benefit of coaching in leadership training, in providing "an opportunity to pause and look at how we do our work ... [since] our request for additional resources is often only part of the answer ... [since] in many instances it is about doing things differently with the resources we have." Another coach spoke of the unrealised potential in coaching: 
The nature of the personal awareness work achieved in developing the competency involves shifts in personal beliefs and attitudes that enable information to be put into action. This learning can be applied in all aspects of the coachee's professional and personal life ... for example: an individual can learn many techniques and approaches about building a team and resolving conflict in a team but unless they have personal courage, creativity and are authentic in their approach, limited outcomes can be achieved. This is the kind of personal resources that coaching develops. It is about marrying the information with the capability of the individual (Coach).

Another summarised the particular function of the coaching process that differentiated it from other interventions:

I think coaching is very apt for the development of [clinical leadership] competencies because it is asking questions that are not obvious and questions that are not anywhere in the literature ... [and] very powerful questions that don't get asked in normal circumstances (Coach).

Some coaches offered best-practice principles in the coaching process: "one of the key principles of coaching is that the focus is on the client's agenda"; "I have to go in more neutral into the sessions as a coach, because I am responding to the client's needs and what they want to focus on." One coach referred to the importance of establishing boundaries in the tri-partite coaching situation, "so as to offset the potential or perceived potential for collusion between the coach and line manager." 
Some coachees spoke about the limitations of coaching, such as the coach's inexperience: "it was her first time to coach, so she is very by the book'. Another recognised that coaching required "a while initially to build up that relationship" and another remarked: "maybe my expectations of her were that she was going to be something much more."

Coaches also reflected on problems and challenges encountered in the coaching process, which included cancelled appointments, geographical distance and lack of time to conduct coaching. One wrote that coachees were "particularly slow in general in making contact with their coach" and another wrote of her disappointment that the coaching relationship "never really materialised." A coach wrote of the challenge of ensuring "that coaching sessions were not used to fire fight challenges encountered in day-to-day clinical experience" and another referred to the challenge of having to address "lots of other things like personal issues, conflict issues that are going on for the coachee." This particular challenge was echoed in coaches' written reflections, which referred to the threat to effective coaching when personal and emotional issues emerged. Encountering such challenges raised issues that were not within the contractual remit or professional competence of the coach: "the personal issues of the individual coachee have to be addressed in the first instance ... in advance of commencing the coaching relationship."

\section{Experiencing action learning}

Non-participant observation of one action learning set enabled the researchers to observe the intervention first hand and yielded a detailed narrative description of the process element of an action learning set, from which the following extract is derived: 
Each participant was afforded an opportunity to reconnect with the goals that they had set for themselves from the previous action learning set meeting ... Each participant participated fully in the action learning set processes, actively listening to each other's case story and asking appropriate, insightful and reflective questions. They afforded each other the space for discussion and appeared to value the contribution of each other. All members affirmed personal goals and openly shared them with the group. Each member gave a commitment to taking action and to giving feedback on the outcomes of this action to the next action learning set meeting.

Pathway participants saw action learning as valuable for its emphasis on action, as a way of finding "some way forward towards [problem] resolution" and of being able to "work on that [issue] and you can bring it back to the next meeting and discuss how you have got on with it." One spoke about this extrinsic value of action learning:

You got to know people [and] you knew that other people had issues just like yourself. And I know you weren't given solutions and nobody was judgemental or whatever, but you felt by discussing things out you were going to get some kind of plan or you might be able to form some kind of plan yourself (Pathway participant).

Action learning sets required good group facilitation in order to develop the group dynamics, promote good participant engagement in learning and problem solving, as one senior nurse put it:

The actual group dynamic itself is incredibly powerful ... [and] there is nowhere to hide. The idea here is that you actually learn something and get something out of it. 
And getting feedback from everybody else when they are in the same boat as you, I think for me, that is the beauty about it. And I think a lot of learning takes place in that situation and under that kind of structure ... and I think it is in the action of the exchange, I think that is where the learning takes place (Pathway participant).

\section{Discussion}

This evaluation study of the pilot of a national clinical leader development project generated rich data to describe participant experiences of interventions that were relatively new in an Irish nursing and midwifery context. It was important to generate data that examined these interventions, their nuances and their contribution at both process and outcomes levels; the study data were sufficiently rich and varied to do this. The use of focus groups, interviews, non-participant observation and mentors' and coaches' reflections represented a form of simultaneous within-method data triangulation (Begley 2006). This use of multiple data sources for the same phenomenon added cumulatively to the data set, provided validation of data and ensured that the limits of each individual method were counteracted by the concurrent use of several methods. Hence, the combined benefits of all enriched the overall quality of evaluative evidence concerning the interventions.

\section{Supports and interventions}

With different expectations for front-line nurses in the context of managed change, there is evidence that organizations are investing in leadership development training for nurses who do not occupy management positions (Wessel-Krejci \& Malin 1997). The Clinical Leader Development Pathway in Ireland reflects this trend in that it is designed for all clinical and 
managerial grades and its interventions are designed with reference to a broad spectrum of grade-specific clinical leadership development need.

Mentors were experienced senior co-workers who shared knowledge, skills and experiences to assist the Pathway participant to develop leadership competencies in a challenging, but supportive way. Coaches were senior personnel outside of the immediate departmental setting who focused on specific development areas and coaching was circumscribed by a finite number of coaching sessions (HSE 2011). In evaluating participant experiences, there was no intention to compare the various interventions, in terms of their relative effectiveness in contributing to leadership development and, unlike other research that indicates the superiority of mentoring over coaching in promoting leadership development (Fielden et al. 2009), the study data did not suggest that one intervention was superior to another. Some senior service managers in the UK have indicated a lack of understanding regarding the processes of mentoring and coaching (Lawrence \& Richardson 2012); however, since the Irish programme made a clear distinction between mentoring and coaching at the process level, Pathway participants in the present study did not appear to have similar misunderstandings. The personal presence of the coach has been shown to be important in achieving the desired outcome of coaching (Ammentorp et al. 2013) and it appears that the present study findings attest to this important aspect of coaching and support the processlevel benefits of coaching (Fielden et al. 2009).

Participants' experiences of the various interventions were overall positive. Experiential approaches to leadership development, such as mentoring and coaching, have been widely supported in the literature (Proctor-Thompson 2008). As an intervention, mentoring has been supported by a number of authors (Day 2001, Bush et al. 2007) and in the present study, 
mentoring was found to be successful at the process level, with many participants experiencing the intervention as positive and supportive and contributing to specific clinical leadership competencies.

While mentoring has been shown elsewhere to be strongly effective on motivation and moderately effective on insight, it may not be as effective as either coaching or action learning in the areas of skill development and real-world practice (Leonard \& Lang 2010). Coaching places individuals into real situations in which they see and experience at first hand the unique problems that occur in their practice (Byrne 2007). Evidence from the present study attests to these action and learning-oriented functions of coaching and to the efforts of coaches in helping coachees to formulate practitioner-generated solutions. Many coaches and Pathway participants spoke of coaching as a way of developing the individual for the role that s/he performed.

Action learning was seen as beneficial in providing opportunities for participants to share experiences of practice problems and find possible solutions to them through group processes. Particular strengths of the intervention were its focus on sharing ideas and concerns about practice and its action orientation in group discussions. Action learning and individual coaching are more strongly effective on outcomes like insight, skill development and real-world practice than the more traditional leadership development approaches (Leonard and Lang 2010).

This study was limited by some of the limitations inherent in the qualitative data collection methods used to generate accounts of the interventions. Aside from non-participant observation of a single action learning set, all other data consisted of oral evidence provided 
in focus group discussions and individual interviews. While focus groups can yield rich data, they can also generate consensual evidence that may not truly capture the particularities of the interventions being evaluated. The use of complementary data from independent sources might have provided more nuanced accounts of the interventions. As was a time-bound study, it was not possible to evaluate the development of mentoring and coaching relationships over time.

\section{Conclusions}

This evaluation study of the programme pilot of a national clinical leadership development programme indicates that coaching, mentoring and action learning interventions used were designed to address a broad spectrum of grade-specific clinical leadership development need. The study points to the intrinsic value of these experiential and action-oriented interventions in clinical leadership development. The time-bound nature of the programme pilot did not permit examination of the relative effectiveness of the various interventions or the longerterm impact on the individuals and the service and further research could examine these aspects in the context of the Irish programme.

\section{Relevance to clinical practice}

The use of interventions that are action oriented and focused on service development, such as mentoring, coaching and action learning, should be incorporated into clinical leadership development programmes. In using these interventions, the focus should be on each participant's role and everyday practice and on helping the participant to develop and demonstrate clinical leadership skills in these contexts. Being quite different to short 
attendance courses, these interventions require longer-term commitment on the part of both individuals and their organisations.

\section{References}

Ammentorp J, Jensen HI and Uhrenfeldt L (2013) Danish health professionals' experiences of being coached: A pilot study. Journal of Continuing Education in the Health Professions $33(1), 41-47$.

Barbour R (2007) Doing Focus Groups. Sage, London.

Begley, CM (1996) Using triangulation in nursing research. Journal of Clinical Nursing 24 (1),122-8.

Berg B.L. (1989) Qualitative Research Methods for the Social Sciences. Allyn and Bacon, New York.

Bush T, Glover D, Harris A (2007) Review of School Leadership Development. University of Warwick for NCSL, Warwick.

Byrne G (2007) Unlocking potential: Coaching as a means to enhance leadership and role performance in nursing. Journal of Clinical Nursing 16 (11), 1987-1988.

Day DV (2001) Leadership development: A review. Leadership Quarterly, 11 (4), 581-613. 
**** (2012) The National Clinical Leadership Development Project Pilot Evaluation Dublin: Health Service Executive/University College Dublin.

Fielden SL, Davidson MJ and Sutherland VJ (2009) Innovations in coaching and mentoring: implications for nurse leadership development. Health Services Management Research 22 (2), 92-99.

Health Service Executive (2012) The National Clinical Leadership Development Framework. Available online at: http://www.hse.ie/eng/about/Who/ONMSD/leadership/ (Retrieved 26 December 2012).

Health Service Executive (2011) Mentoring for the National Clinical Leadership Development Pathway: Mentor Guide. Health Service Executive, Dublin.

Krueger RA, Casey MA (2009) Focus Groups: A Practical Guide for Applied Research. Sage, London.

Lawrence N and Richardson J (2012) To explore and understand the leadership experiences of modern matrons, within an acute NHS Trust. Journal of Nursing Management Available online at: http://onlinelibrary.wiley.com/doi/10.1111/j.1365-2834.2012.01432.x/full (Retrieved 22 April 2013).

Leonard HS \& Lang F (2010) Leadership development via action learning. Advances in Developing Human Resources 12, 225-240. 
Lunn C, MacCurtain S and McMahon J (2008) Clinical Leadership Pilot Evaluation Report. Health Service Executive, Dublin.

Newell R. and Burnard P. (2006) Research for Evidence-Based Practice Blackwell, Oxford.

Proctor-Thomson SB (2008) Constellations or stars? What is being developed in leadership development? Lancaster University Management School, Centre for Excellence in Leadership (CEL), Lancaster.

Reid Ponte P, Gross A, Gallante A \& Glazer G (2006) Using an executive coach to increase leadership effectiveness. Journal of Nursing Administration 36 (6), 1-6.

Royal College of Nursing (1997) Ward Leadership Project: A journey to patient centre Leadership, London: RCN.

Wessel-Krejci JW \& Malin S (1997) Impact of leadership development on competencies. Nursing Economics, 15, 235-41. 